\title{
Challenging the Testing Protocol of the BOD POD, A Repeated Measures Design
}

\author{
Ahmed Elokda, PhD, PT, CLT, CEEAA, FAACVPR ${ }^{*}$, Sarah Bengtson, DPT ${ }^{2}$, Sierra Griffin, DPT ${ }^{3}$, Eric Shamus, PhD, \\ DPT $^{4}$ \\ ${ }^{1 *}$ Department of physical therapy management of Internal Medicine disorders, Cairo University, Cairo, Egypt. \\ ${ }^{2,3}$ Physical Therapist, Fort Myers, FL, USA. \\ ${ }^{4}$ Department of Rehabilitation Sciences, Marieb College of Health \& Human Services, Florida Gulf Coast University, Fort \\ Myers, FL, USA.
}

\section{Article Details \\ Article Type: Research Article \\ Received date: $06^{\text {th }}$ June, 2020 \\ Accepted date: $06^{\text {th }}$ July, 2020 \\ Published date: $07^{\text {th }}$ July, 2020}

"Corresponding Author: Ahmed Elokda, PhD, PT, CLT, CEEAA, FAACVPR, Department of Rehabilitation Sciences, Marieb College of Health \& Human Services, Florida Gulf Coast University, 10501 FGCU Boulevard South, Fort Myers, FL 33965-6565, United States. E-mail: aelokda@,fgcu.edu

Citation: Elokda, A., Bengtson, S., Griffin, S., \& Shamus, E. (2020). Challenging the Testing Protocol of the BOD POD, A Repeated Measures Design. J Rehab Pract Res 1(1):107. https://doi.org/10.33790/jrpr1100107

Copyright: (C2020, This is an open-access article distributed under the terms of the Creative Commons Attribution License 4.0, which permits unrestricted use, distribution, and reproduction in any medium, provided the original author and source are credited.

\section{Abstracts}

The BOD POD uses air displacement plethysmography to determine an individual's body composition of fat and fat-free mass. There were no studies found examining the consumption of fluids and pre-urination prior to body composition testing utilizing the BOD POD. The objective was to determine if the BOD POD protocol, by Life Measurement, Inc., needs to be followed in its entirety to ensure validity of body composition results. Thirty-two Division 1 college soccer athletes were recruited for this research study at the human performance lab. In a repeated measures design, four separate measurements of body composition were taken: preurination, standard Life Measurement protocol test re-test, and consumption of water equal to $10 \%$ of their body weight in ounces. A Pearson product moment correlation between the second condition (post urination) and the third condition (post urination retest) signified a good to excellent relationship between the standard test and the retest conditions $(0.977, \mathrm{p}<.001)$. A MANOVA analysis was performed comparing pre-urination and post-urination test/retest indicated that not urinating prior to testing had no significant effect on body composition measurements $(p>.05)$. Consumption of water did have a significant effect on the results of the body composition measurements. It was found that post water consumption, male participants' measurement of fat mass on average had a significant difference of $0.6 \%$ and female participants' fat mass measurements on average had a difference of $1.4 \%, \mathrm{p}<.001$. Results did not support the need to urinate prior to BOD POD testing while supporting the need to refrain from water consumption directly prior to testing.

Keywords: Body Composition, Metabolic Testing, Body Fat, Body Mass

\section{Strengths and limitations of this study}

- This study utilized state of the art, plethysmography to determine an individual's body composition.

- This study was a repeated measures design, using the same subjects as their control.

- This study did not measure the exact volume of urine in the bladder before and after voiding.

- This study relies on participants to understand and adhere to the
BOD POD protocol established and distributed to the coaching staff prior to testing, without means for verification of participant adherence other than signed consent.

\section{Introduction}

Air displacement plethysmography (ADP) is a method of assessing body composition utilized since the 1990's. The use of ADP has grown immensely due to its efficiency and ability to accurately measure body composition as compared to the gold standard of hydrostatic weighing [1]. In a clinical setting, measurement of body mass index (BMI) for different populations may overestimate or underestimate fat composition, thus making it an unreliable source of measurement. Use of ADP can help narrow the level of unreliability of that noted by body mass index (BMI) measurements, but how accurate is ADP, in particular the BOD POD?

The BOD POD has been demonstrated to be a valid and reliable standard of measurement for body composition and assessing fat mass versus fat free mass [2], comparable to the gold standard of hydrostatic weighing within 1\% [3]. The BOD POD has also been demonstrated to be reliable with same day testing of subjects, demonstrating no statistically significant differences between body fat percentages for subjects tested within the same day [4].

In roughly 5 to 10 minutes, the BOD POD is capable of assessing body composition, fat mass, fat free mass, and measuring lung volume within the intrathoracic cavity. ADP uses gas laws to describe the inverse relationship between pressure and volume in two enclosed chambers. Via the SIRI or Shutte equations, the BOD POD uses measurements of density to determine fat mass and fat free mass percentages.[5] Once this information is obtained, various practitioners are able to take those measurements and apply them as needed.

Most of the current research for BOD POD testing has focused on challenging the BOD POD protocol by having participants hold various amounts of fluids, various types of fluids [6], or changing clothing type worn [5], but none have considered the direct consumption of fluids or the effects voiding may have on measurements of body composition via BOD POD testing. Other research has focused on the compensatory techniques to altering body composition results in elite athletes, demonstrating that air trapped 
against the subjects can alter results [7].

The practicality for ADP to come into use in a clinical setting requires its parameters to be questioned and tapered to fit the population as a whole. Within the population at large, it may be unrealistic for some groups, such as those with diabetes, to refrain from water intake for more than four hours. For this reason, it is necessary to challenge this aspect of the manufacturer's protocol in order to make BOD POD testing more feasible for a greater number of diverse clients. The purpose of this study was to determine if the BOD POD protocol, as set forth by Life Measurement, Inc., needs to be followed in its entirety to ensure validity of body composition results. Thus, the hypothesis was that breaking the established protocol would not have any statistically significant effects on body composition results.

\section{Methods}

\section{Experimental Approach to the Problem}

All subjects acted as their own control in a repeated measures design, in which subjects performed all 4 tests with the BOD POD system, pre-urination, standard protocol test, standard protocol retest, and post water consumption. Each participant was allotted the same amount of time between testing sessions which consisted of 5 minutes between pre-urination and standard protocol test, immediate testing between standard protocol testing and standard protocol retesting, and 10 minutes between standard protocol retest and post water consumption. Testing was conducted in the Sports Performance Lab during unoccupied hours, at various times of the day at XXXXXXX. Between pre-urination and standard protocol testing, subjects were asked to urinate using the lab facilities, exclusive to study participants. Subjects were asked to consume a measured amount of water, equaling $10 \%$ of their body weight in fluid ounces, prior to the final test. Variables of interest included body composition measurements, fat mass and fat free mass, of preurination and post water consumption in comparison to standard protocol test-retest results. Analyses were conducted to determine between-trial differences in these variables.

\section{Subjects}

A total of 32 Division 1 athletes participated in the BOP POD research study, $46 \%$ male and $54 \%$ female. Access to Division 1 athletes was obtained through XXXXX Athletic Department. Coaches for each team were contacted via e-mail and were given the option to allow their athletes to participate in the research study, as BOD POD/body composition testing is a part of their pre-season fitness testing. Out of 16 Division 1 coaches contacted, two coaches (men's and women's soccer) responded and gave permission for their athletes to participate in the BOD POD research study. All participants were informed of the testing protocol, what was expected of them during testing, and that all data collected for this study would be anonymous. Demographic data were analyzed using descriptive statistics including frequency counts, percentages, mean, and measures of dispersion. Thirty-two Division 1 male (15) and female (17) soccer athletes from XXX were recruited for this research study. Male soccer players weighed 162.6 pounds $(+19.03)$ and female soccer players weighed 133.8 pounds $(+$ 10.38). All athletes were between 18 and 22 years old (Male $+1.37 \&$ Female +1.17$)$. The BOD POD system utilizes two different ethnic descriptors in terms of body composition equation used, thus of the 32 Division 1 soccer athletes, 6 were of African descent (5 male, 1 female) and 26 were considered general population (10 male, 16 female). Participants were included if they were a Division 1 athlete at XXXX, and they were at least 18 years of age. Exclusion from the study would have occurred if any participant had not followed the BOD POD pre-testing conditions as set forth by Life Measurement. No participants were excluded from the study from the inclusion/ exclusion criteria. Prior to data collection, the study protocol and all related documents, including informed consent, were submitted and approved through the Institutional Review Board (IRB) at XXXX. Approval and oversight of the IRB allowed for protection of human subjects and ensured compliance with the policies and procedures established by XXXX, as well as any additional legal or ethical concerns. To ensure anonymous collection of data, all participants were given a numerical identifier. Prior to testing, participants were asked to sign informed consent forms which included signed understanding of testing protocol and verification that protocol was followed prior to attendance.

\section{Patient Involvement}

Patients were not involved in this study.

\section{Procedures}

Each participant was subjected to BOD POD testing under four different conditions to determine if any statistically significant changes were noted in body composition measurements.

1. pre-urination

2. standard test

3. standard re-test

4. post water consumption

Per Life Measurement protocol, participants were asked to refrain from eating, drinking, and exercising 3 to 4 hours prior to their scheduled testing time. Participants were also asked to refrain from urinating 2 hours prior to testing. Based on the literature regarding the average filling rates of the bladder, it can be inferred that the individuals in this study had roughly $168 \mathrm{~mL}$ volume in their bladders at the time of the first measurement. This value was determined based on normal levels of hydration, and the average rate of filling for the bladder, $1.4 \mathrm{~mL} / \mathrm{min}$ [8], and the time participants were asked to refrain from urinating prior to testing. Upon arrival, and prior to each measurement, subjects were asked to change into tight-fitting compression clothing and to remove all jewelry. Subjects wore a tight-fitting swim cap during ADP to minimize the effect of the hair on body volume assessment [9]. Body mass was first measured to the nearest $0.02 \mathrm{~kg}$ on a calibrated electronic scale. Each subject was then asked to sit in the BOD POD for body volume measurement. Subjects were instructed to sit quietly with an erect posture and normal respiration, with their hands folded in their laps and their feet placed on the floor of the device. A minimum of two 50-second tests were conducted to ensure reliability of measurements. The body volume measurement was repeated if the 2 measures were not within $150 \mathrm{~mL}$ of each other [9]. The first measurement was taken prior to urination. Participants were then asked to urinate between the first and second tests. The second and third tests consisted of test-retest measurements, in which participants were following the established manufacturer's testing protocol for the BOD POD. Finally, a fourth measurement was performed after participants had consumed a predetermined amount of water ( $10 \%$ of their body weight in ounces) as measured during the second and third tests (test and retest).

\section{Statistical Analyses}

A Pearson's and Spearmen's Correlation test was used to run a power analysis to determine the appropriate sample size needed to achieve statistical significance. With a power level set at .80 , correlation of .50 , and significance of .50 , it was determined that atleast 29 participants were needed to achieve statistical significance. A MANOVA was performed to test each variable against the three others. Pairwise comparisons were used to identify which conditions were responsible for the detected variation in outcomes at a level of statistical significance of $p<0.05$. The first pairwise comparison related baseline test-retest body composition measurements to pre voiding measurements and the second compared test-retest body composition measurements to measurements taken post water consumption. A $95 \%$ confidence interval was used during the testing parameters. A Wilks' Lambda indicates there was a statistically significant difference $(p$ value $<.001)$ between all testing variables, with 3 degrees of freedom. Further analysis was performed using a pairwise comparison of the first condition (pre-urination), second condition 
(post urination), third condition (post urination retest) and the fourth condition (water consumption/post hydration) resulting in three degrees of freedom for each condition.

\section{Results}

\section{Test-Retest Validity}

A Pearson moment correlation was calculated between the second condition (post urination) and the third condition (post urination retest). This test yielded a Pearson moment correlation value of 0.977 , signifying a good to excellent relationship between the standard test and the retest.

\begin{tabular}{|l|l|l|l|}
\hline \multirow{4}{*}{ Standard Protocol } & Pearson Correlation & Standard Protocol & Retest Protocol \\
\cline { 2 - 4 } & Sig. (2-tailed) & 1 & $.977^{* *}$ \\
\cline { 2 - 4 } & $\mathrm{N}$ & 32 & .000 \\
\hline \multirow{5}{*}{ Retest Protocol } & Pearson Correlation & $.977^{* *}$ & 32 \\
\cline { 2 - 4 } & Sig. (2-tailed) & .000 & 1 \\
\cline { 2 - 4 } & $\mathrm{N}$ & 32 & 32 \\
\hline \multirow{2}{*}{$* *$ Correlation is significant at the 0.01 level (2-tailed). } \\
\hline
\end{tabular}

\begin{tabular}{|l|l|l|l|l|l|l|l|l|}
\hline & Value & $\mathrm{F}$ & $\begin{array}{c}\text { Hypothesis } \\
\text { df }\end{array}$ & $\begin{array}{c}\text { E r r o r } \\
\text { df }\end{array}$ & Sig. & $\begin{array}{c}\text { Partial Eta } \\
\text { Squared }\end{array}$ & $\begin{array}{c}\text { Noncent. } \\
\text { Parameter }\end{array}$ & $\begin{array}{c}\text { Observed } \\
\text { Power }\end{array}$ \\
\hline Pillia's Trace & 0.408 & $6.658^{\mathrm{a}}$ & 3.000 & 29.000 & 0.001 & 0.408 & 19.974 & .953 \\
\hline $\begin{array}{l}\text { Wilks' } \\
\text { Lambda }\end{array}$ & 0.592 & $6.658^{\mathrm{a}}$ & 3.000 & 29.000 & 0.001 & 0.408 & 19.974 & .953 \\
\hline $\begin{array}{l}\text { Hotelling's } \\
\text { Trace }\end{array}$ & 0.689 & $6.658^{\mathrm{a}}$ & 3.000 & 29.000 & 0.001 & 0.408 & 19.974 & .953 \\
\hline $\begin{array}{l}\text { Roy's } \\
\text { Largest root }\end{array}$ & 0.689 & $6.658^{\mathrm{a}}$ & 3.000 & 29.000 & 0.001 & 0.408 & 19.974 & .953 \\
\hline
\end{tabular}

First condition to second condition test: The first condition (preurination) compared to the standard test (post-urination) yielded a Pearson moment correlation value of 0.322 . This analysis compared the pre-urination test to urinating then testing. There was no significant difference between these tests, $\mathrm{p}>.05$. This compared the first condition to the second condition performed. First condition to third condition test: The first condition (pre-urination) compared to the standard re-test (post-urination) yielded a Pearson moment correlation value of 0.452 . In this analysis, the first pre-urination condition was compared to a retest after urination. The results also showed no significant difference between the first condition and the third condition performed. This also demonstrated no significant difference on retest results as there was no differing factors between the second and the third test, $\mathrm{p}<.05$.

First condition to fourth condition test: The first condition (pre-urination) compared to the fourth condition (water consumption/ post hydration) yielded a Pearson moment correlation value of 0.044 . This showed a significant difference that adding in hydration significantly changed the results of the test, $\mathrm{p}<.05$.

Second condition to fourth test: The second condition (posturination) compared to the fourth test (water consumption/post hydration) yielded a Pearson moment correlation value of 0.002 . This showed a significant difference that adding in hydration significantly changed the results of the test, $\mathrm{p}<.05$.

Finally, the third condition to the fourth condition test: the third condition (post-urination retest) compared to fourth condition (water consumption/post hydration) yielded a Pearson moment correlation value of 0.000 . This showed a significant difference that adding in hydration significantly changed the results of the test, $\mathrm{p}<.05$.

\begin{tabular}{|l|l|l|l|l|l|l|}
\hline & & & & \multicolumn{3}{|c|}{$\begin{array}{l}\text { 95\% Confidence Interval for } \\
\text { Difference }^{\mathrm{b}}\end{array}$} \\
\hline (I) Condition & (J) Condition & $\begin{array}{l}\text { Mean } \\
\text { Difference } \\
\text { (I - J) }\end{array}$ & Std. Error & Sig. ${ }^{\text {b }}$ & Lower Bound & Upper Bound \\
\hline 1 & 2 & 0.303 & 0.301 & 0.322 & -0.311 & 0.917 \\
\hline & 3 & 0.228 & 0.299 & 0.452 & -0.382 & 0.838 \\
\hline & 4 & -0.728 & 0.347 & 0.044 & -1.435 & -0.021 \\
\hline & 1 & -0.303 & 0.301 & 0.322 & -0.917 & 0.311 \\
\hline & 3 & -0.075 & 0.205 & 0.717 & -0.493 & 0.343 \\
\hline
\end{tabular}

Table. 3 To be Cont........... 


\begin{tabular}{|l|l|l|l|l|l|l|}
\hline 3 & 1 & -0.228 & 0.299 & 0.452 & -0.838 & 0.382 \\
\hline & 2 & 0.075 & 0.205 & 0.717 & -0.343 & 0.493 \\
\hline & 4 & -0.956 & 0.208 & 0.000 & -1.38 & -0.532 \\
\hline 4 & 1 & 0.728 & 0.347 & 0.044 & 0.021 & 1.435 \\
\hline & 2 & 1.031 & 0.296 & 0.002 & 0.427 & 1.635 \\
\hline & 3 & 0.956 & 0.208 & 0.000 & 0.532 & 1.38 \\
\hline
\end{tabular}

\section{Discussion}

It is thought that excess fluids in the body will be inappropriately categorized as fat mass when using the BOD POD for body composition measurements. The Life Measurement protocol requires participants to urinate prior to testing in attempts to remove any excess fluids that may skew the results of the fat mass versus fat free mass compositions. All participants in this study acknowledged that they followed the no urination for two hours prior to testing requirement. The major finding of the present study was that breaking protocol for BOD POD testing resulted in a significant difference on body composition measurements. Compared with standard protocol testing, consumption of water provided significantly different results in body composition testing ( $\mathrm{p}<.05)$, meanwhile voiding before testing did not have a significant difference $(p>.05)$. This is the first study, to our knowledge, that examined the consumption of water and its effects on body composition as measured by the BOD POD. Carvennec, et al. [6] assessed the influence of consumption/hydration status on body composition as measured by the BOD POD in obese individuals. Ten test subjects were subjected to body composition testing via the BOD POD. Initial body composition measures were taken along with subsequent testing measurements while subjects held a water bottle, bottle of oil, and bottle of combined water and oil. Results concluded that water loads caused an underestimation of fat-free mass while also causing an overestimation $(0.8 \%, p<0.001)$ of fat mass. In the current research study, participants consumed $10 \%$ of their body weight in fluid ounces of water and were retested and found to have an over estimation of fat mass and an underestimation of fat-free mass. Although this study involved the consumption of fluids versus simply holding them, it still verifies that addition of water to BOD POD testing, in one form or another, creates an over estimation of fat mass.

Another study conducted by Utter, et al. [10] measured the differences between BOD POD and hydrostatic weighing (HW) in wrestlers who were well hydrated and acutely dehydrated. Although they found there to be no statistically significant difference between BOD POD testing and hydrostatic weighing for either hydrated or acutely dehydrated individuals ( $p>.05$ ), they did find that all methods of body composition measurements showed a significant decrease in fat free mass when comparing the hydrated to the dehydrated state. Although this Another study conducted by Utter et al., [10] measured the differences between BOD POD and hydrostatic weighing (HW) in wrestlers who were well hydrated and acutely dehydrated. Although they found there to be no statistically significant difference between BOD POD testing and hydrostatic weighing for either hydrated or acutely dehydrated individuals $(p>.05)$, they did find that all methods of body composition measurements showed a significant decrease in fat free mass when comparing the hydrated to the dehydrated state. Although this study set out to test BOD POD verses hydrostatic weighing, it is worth mentioning that these results indicate hydration status does play a significant role in the outcome of body composition as measured via ADP and the BOD POD, whereas bladder contents do not play a significant role in body composition measurements as indicated by the current study and are not misappropriated as fat or fat free mass.
Heiss, et al. [11] conducted a study to determine the effect liquid and a semisolid meal have on body composition results as measured via ADP, Bone densitometry, also called dual-energy x-ray absorptiometry (DEXA), and Bio-Electrical Impedance Anaylsis (BIA). Thirty-two participants were measured both before and after consumption of $350-\mathrm{mL}$ Gatorade or cereal with milk. They found that with all three types of measurements, there was no significant difference in percent body fat measured before or after the food or fluid load by DEXA, BIA, or ADP. This differs from the study conducted by Carvennec, et al. [6] and the present research study which found that direct consumption of fluid prior to BOD POD testing, where fluid results in an overestimation of fat mass.

To parallel the results of the current study, Vukovich and Peeters [12], found that the consumption of $500 \mathrm{~mL}$ of water resulted in a significant increase in body mass and body volume $(\mathrm{p}<0.05)$. According to the ADP analysis most of this change was interpreted as fat mass (FM) $(0.35 \mathrm{~kg})$ while the remaining change was interpreted as fat-free mass (FFM) $(0.15 \mathrm{~kg})$. Five hundred $\mathrm{mL}$ equates to roughly 16 fluid ounces, and in the present research study consuming $10 \%$ of water in fluid ounces, 11.9 to $20.3, \mathrm{SD}+2.08$, of each athlete's body weight produced a significant difference.

The standard pretesting protocol set forth by Life Measurement asks participants to refrain from drinking fluids four hours prior to BOD POD measurements being taken. This study found there to be a statistically significant difference between the test-retest and the post water consumption conditions $(\mathrm{p}<.05)$. Consuming $10 \%$ of an individual's body weight in water seems to have a significant impact on a participant's body composition outcomes. The difference between the test-retest fat mass percentages was $0.2 \%$ for males and $0.3 \%$ for females. It was found that post water consumption, male participants' measurement of fat mass on average had a difference of $0.6 \%$ and female participants' fat mass measurements on average had a difference of $1.4 \%$. Since reliability was verified between test-retest measures, this was found to be statistically significant, $(p<.05)$.

A limitation to the study was relying on participants to understand and follow the BOD POD protocol established and distributed to the coaching staff prior to testing, without means for verification of participant adherence other than signed consent. Also, without additional measures, it was difficult to determine a relationship between BOD POD testing pre- and post-urination, and what effects this may have on body composition, as the exact volume of urine in the bladder before and after voiding is unknown. Ideally, the use of diagnostic ultrasound to measure the amount of CC's of urine in the bladder under each condition would be optimal to better represent differences in body composition measurements obtained before and after urination using BOD POD testing. Instead, this study utilized a calculated estimation of urine content based on the rate of bladder filling and the time from last urination to the time of the first BOD POD measurement. Any time a calculated estimation is utilized, there is the potential for error decreasing reliability of measurements and data collection.

It appears that one aspect (hydration) of the BOD POD protocol has statistical significance while one aspect did not (urination). Minimal differences existed between pre-urination measurements and 
post-urination, standard test and retest measurements, and it should be noted that body weight was taken into account with each body composition measurement. Hypothetically, extra fluid content in the body has the potential to alter body composition measurements; therefore, it would be assumed that removing all extra fluid would minimize false variations. However, this study found that the contents of the bladder had no significant effects on body composition measurements, refuting the need to empty the bladder prior to testing.

On the other hand, consuming $10 \%$ of a participant's' weight in ounces of water did result in a statistically significant difference in measurements. Fluid consumption skewed the fat mass to fat free mass distribution measurements, creating a false increase in fat mass and a false decrease in fat free mass. This disproved our hypothesis that fluid consumption would have no effect on the body composition measurements using ADP and verifies the need to limit fluid intake prior to testing.

\section{Practical Applications}

When performing BOD POD tests in a clinical setting, this study did not support the need to empty the bladder prior to testing while supporting the need to refrain from water consumption directly prior to testing. This is useful to the clinician in providing instructions to patients that may have difficulty refraining from urination for longer than 2 hours prior to testing, or who may have difficulty urinating directly preceding testing. The clinician should, however, continue to encourage patients to abstain from drinking fluids prior to BOD POD testing, as this has been demonstrated to significantly impact the results of the body composition analysis.

Conflicts of interest/Competing interests: Authors report no conflict or competing interest.

Funding: This research received no specific grant from any funding agency in the public, commercial or not-for-profit sectors.

This project was approved by the IRB of the institution where research was performed.

\section{References}

1. Fields, D.A., Higgins, P.B., Radley, D. (2005). Air-displacement plethysmography: here to stay. Curr Opin Clin Nutr Metab Care, 8(6):624-9.

2. Biaggi, R.R., Vollman, M.W., Nies, M.A., et al. (1999). Comparison of air-displacement plethysmography with hydrostatic weighing and bioelectrical impedance analysis for the assessment of body composition in healthy adults. Am J Clin Nutr, 69(5):898-903.

3. Fields, D.A., Hunter, G.R., Goran, M.I. (2000). Validation of the BOD POD with hydrostatic weighing: influence of body clothing. Int J Obes Relat Metab Disord, 24(2):200-5.

4. Anderson, D.E. (2007). Reliability of air displacement plethysmography. J Strength Cond Res, 21(1):169-72.

5. Siri, W.E. (1993). Body composition from fluid spaces and density: analysis of methods. Nutrition, 9(5):480-91.

6. Carvennec, M., Fagour, C., Adenis-Lamarre, E., et al. (2007). Body composition of obese subjects by air displacement plethysmography: the influence of hydration. Obesity (Silver Spring), 15(1):78-84.

7. Tegenkamp, M.H., Clark, R.R,, Schoeller, D.A., et al. (2011). Effects of covert subject actions on percent body fat by air-displacement plethsymography. J Strength Cond Res, 25(7):2010-7.

8. Lee, S.W., Kim, J.H. (2008). The significance of natural bladder filling by the production of urine during cystometry. Neurourol Urodyn, 27(8):772-4.

9. Dempster, P., Aitkens, S. A new air displacement method for the determination of human body composition. Med Sci Sports Exerc, 27(12):1692-7.
10. Utter, A.C., Goss, F.L., Swan, P.D., et al. (2003). Evaluation of air displacement for assessing body composition of collegiate wrestlers. Med Sci Sports Exerc, 35(3):500-5.

11. Heiss, C.J., Naylor, J., Bronco, K.M., et al. (2008). A small food or fluid load has no effect on body composition measured by 3 different methods. Topics in Clinical Nutrition, 23(3):229-33.

12. Vukovich, M.D., \& Peeters, B.M. (2003). Reliability of airdisplacement plethysmography in detecting body composition changes after water ingestion and after creatine supplementation. J Exerc Physiology Online, 6:115-22. 